\title{
Criminologie
}

\section{Comment mesurer la délinquance des toxicomanes adultes? Une comparaison empirique de la validité des registres de police et des sondages de délinquance autoreportée}

\section{Marcelo F. Aebi}

Volume 35, numéro 1, printemps 2002

L'âge et la question criminelle

URI : https://id.erudit.org/iderudit/027516ar

DOI : https://doi.org/10.7202/027516ar

Aller au sommaire du numéro

Éditeur(s)

Les Presses de l'Université de Montréal

ISSN

0316-0041 (imprimé)

1492-1367 (numérique)

Découvrir la revue

Citer cet article

Aebi, M. F. (2002). Comment mesurer la délinquance des toxicomanes adultes ? Une comparaison empirique de la validité des registres de police et des sondages de délinquance autoreportée. Criminologie, 35(1), 107-131.

https://doi.org/10.7202/027516ar

\section{Résumé de l'article}

Cet article se base sur une analyse comparative des interpellations enregistrées par la police et des délits admis par un échantillon de toxicomanes adultes dans le cadre d'un sondage de délinquance autoreportée. La moyenne d'âge des membres de l'échantillon ( $\mathrm{N}=491$ dont 135 femmes) est de 31 ans. Il s'agit de toxicomanes qui étaient très engagés dans la délinquance avant de participer aux essais suisses de prescription d'héroïne. Différents concepts de validité sont testés, à savoir La validité de contenu (qui comprend la validité apparente et la validité logique) et la validité concourante (qui comprend la validité pour groupes différents et la validité corrélationnelle). L'analyse montre que ce n'est pas tellement le nombre de délits commis qui détermine le risque de se faire interpeller par la police, mais plutôt la diversité et la gravité de ces délits. Elle montre également que, dans le contexte étudié, le sondage de délinquance autoreportée s'avère être un indicateur valide de La criminalité. En fait, le sondage offre plus d'information et permet de déceler un plus grand nombre de délin- quants et de délits que les données de police. 


\title{
Comment mesurer la délinquance des toxicomanes adultes ?
}

\section{Une comparaison empirique de la validité des registres de police et des sondages de délinquance autoreportée}

\author{
Marcelo F. Aebi \\ Professeur remplaçant \\ Institut de police scientifique et de criminologie \\ Université de Lausanne \\ Professeur visitant \\ Institut Andalou de criminologie \\ Université de Séville \\ aebi@us.es
}

RÉSUMÉ - Cet article se base sur une analyse comparative des interpellations enregistrées par la police et des délits admis par un échantillon de toxicomanes adultes dans le cadre d'un sondage de délinquance autoreportée. La moyenne d'âge des membres de l'échantillon ( $N=491$ dont 135 femmes) est de 31 ans. Il s'agit de toxicomanes qui êtaient très engagés dans la délinquance avant de participer aux essais suisses de prescription d'héroïne. Différents concepts de validité sont testés, à savoir la validité de contenu (qui comprend la validité apparente et la validité logique) et la validité concourante (qui comprend la validité pour groupes différents et la validité corrélationnelle). L'analyse montre que ce n'est pas tellement le nombre de délits commis qui détermine le risque de se faire interpeller par la police, mais plutôt la diversitê et la gravité de ces délits. Elle montre également que, dans le contexte étudié, le sondage de délinquance autoreportée s'avère être un indicateur valide de la criminalité. En fait, le sondage offre plus d'information et permet de déceler un plus grand nombre de délinquants et de délits que les données de police.

ABSTRACT - This paper is based on a comparison of self-reported delinquency and police recorded contacts for a sample of adult drug-addicts. The average age of the sample 
( $N=491$ of which 135 are females) is thirty-one. The members of the sample were heavily involved in delinquency before entering a heroin prescription program in Switzerland. Different concepts of validity are tested, namely content validity (including face validity and logical validity) and concurrent validity (including known-group validity and correlational validity). The results show that the risk of having a police contact is explained better by the variety and the seriousness of the offences than by the number of offences. They also show that the self-report method can produce valid results in this particular context. Moreover, self-reports seem to measure the amount and patterns of delinquent behavior more accurately than official data.

\section{Introduction}

Il est bien connu que les indicateurs de la criminalité présentent des problèmes de fiabilité et de validité qui les empêchent de mesurer de manière précise le comportement délinquant. Dans ce contexte, la comparaison des délits enregistrés par différents indicateurs durant la même période et pour le même groupe de personnes s'avère particulièrement intéressante, parce qu'elle permet d'apprécier les convergences et les divergences des indicateurs comparés. Néanmoins, on trouve relativement peu de recherches empiriques sur ce sujet, et encore moins avec des échantillons composés d'adultes ${ }^{1}$. Ce manque de recherches pourrait s'expliquer par le fait qu'en multipliant les sources des données, on multiplie également le coût de la recherche. D'autre part, les restrictions imposées par les lois sur la protection des données personnelles rendent difficiles la récolte et la conservation des informations individuelles indispensables pour procéder à une telle recherche (Killias, 2000).

Dans cette perspective, l'évaluation des programmes suisses de prescription d'héroïne sous l'angle de la délinquance - confiée à l'Institut de police scientifique et de criminologie (IPSC) de l'Université de Lausanne - offre une excellente opportunité de procéder à une telle comparaison. En effet, afin de mesurer les effets de ces programmes sur la délinquance des toxicomanes traités, plusieurs indicateurs de la criminalité ont été retenus. Ainsi, les participants ont répondu à des sondages de délinquance autoreportée et de victimisation au moment de leur admission dans le programme et à des intervalles de 6 mois par la suite ; nous avons par la suite relevé leurs condamnations judiciaires inscrites au Casier judiciaire central ainsi que les interpellations enregistrées par

1. Pour une revue de la littérature, voir Aebi (1999). 
les corps de police des cantons et des villes où se déroulaient ces programmes. Ces données ont été recueillies entre 1995 et 1996.

\section{Méthodologie}

Étant donné que nous les avons présentées ailleurs, nous ne nous attarderons pas ici sur les particularités des indicateurs de la criminalité utilisés (voir Aebi, 2000), ni sur les caractéristiques des programmes suisses de prescription d'héroïne (voir Aebi et al., 1999 ; Killias et al., 1999). De même, nous limiterons notre présentation à la comparaison des sondages de délinquance autoreportée et des interpellations enregistrées par la police ${ }^{2}$ durant les six mois antérieurs à l'admission des participants dans le programme (ci-après "période avant l'admission ») et durant les douze mois postérieurs à ladite admission (ci-après "période après l'admission "). Néanmoins, notons que pour être admis dans un programme de prescription d'héroïne, il fallait être âgé d'au moins vingt ans, être dépendant de l'héroöne depuis au moins deux ans, avoir essayé des traitements conventionnels sans succès et présenter des dommages à la santé ou sur le plan social dus à la consommation de drogue. Ces programmes se sont déroulés dans dix-huit centres de traitement ambulatoire (établis dans différentes villes suisses) auxquels les personnes devaient se rendre entre une et trois fois par jour pour recevoir l'hérö̈ne qu'elles devaient consommer sur place et sous contrôle médical. L'équipe de collaborateurs de chacun de ces centres (où l'on trouvait, entre autres, des médecins, des psychologues et des assistants sociaux) s'est chargée du recrutement des toxicomanes lequel s'est déroulé sur une assez longue période de temps (c'est la raison pour laquelle le temps d'observation (avant et après l'admission) est calculé de manière individuelle).

Pour la période avant l'admission dans le programme, l'échantillon étudié est composé de 491 personnes, dont 135 femmes. La moyenne d'âge est de 31 ans. Ce dernier point est particulièrement important, parce que la plupart des chercheurs s'accordent à penser que les sondages de délinquance autoreportée constituent un indicateur peu valide pour mesurer la criminalité des adultes, sauf si l'échantillon est

2. Pour une comparaison des autres indicateurs utilisés, voir Aebi (1999). 
composé d'adultes incarcérés (Junger-Tas et Marshall, 1999³). En retour, des recherches indiquent que les jeunes délinquants connus de la police ou des tribunaux sont particulièrement disposés à avouer leurs délits, et encore plus s'ils savent que leurs antécédents policiers et judiciaires seront consultés (voir Blackmore, 1974 et Farrington, 1977 ; 1989 ; ainsi qu'un traitement plus détaillé de cette question dans Aebi 1999 : 162ss). Ceci est également important dans le cadre de notre recherche autant parce que les participants avaient donné leur accord pour que leurs antécédents soient consultés que parce que la plupart avait đéjà eu des contacts avec le système de justice pénale.

En général, les membres de l'échantillon était très impliqués dans la délinquance. En effet, $74 \%$ d'entre eux avaient déjà été condamnés par un tribunal, $87 \%$ avaient été interpellés par la police au moins une fois durant les sept dernières années en tant que suspects de la commission d'un délit, $95 \%$ avouaient avoir commis dans le courant de leur vie au moins l'un des délits inclus dans le sondage, et $69 \%$ avouaient avoir commis au moins l'un de ces délits dans le courant des six derniers mois. La combinaison de ces trois indicateurs de la criminalité montre que seul $1 \%$ de l'échantillon est classé comme non délinquant par tous les trois. De même, signalons que d'après les registres de police et les sondages de délinquance autoreportée, les délits les plus fréquemment commis par les membres de l'échantillon étaient le trafic de drogue et le vol à l'étalage.

En ce qui concerne les indices de la délinquance établis à partir de ces indicateurs, nous nous intéresserons notamment à la prévalence, à l'incidence et à la diversité de la délinquance. Dans le cadre de notre recherche, et en prenant comme point de départ le sondage de délinquance autoreportée, les définitions de ces indices sont les suivantes:

a) la prévalence exprime le pourcentage de personnes de l'échantillon qui ont admis avoir commis au moins un délit durant la période considérée ;

b) l'incidence exprime le nombre moyen de délits avoués par les membres de l'échantillon durant la période considérée ;

c) la diversité exprime l'étendue de la gamme de délits avoués par les membres de l'échantillon durant la période considérée. Pour établir

3. Les problèmes de validité, tant pour les adultes (Reuband, 1989) que pour les lycéens (Erickson et Empey, 1963), semblent être liés au concept de " désirabilité sociale " (social desirability) qui mènerait ces groupes de personnes à se présenter comme des individus bien intégrés dans la société. Ainsi, étant donné que la délinquance est un comportement contraire aux normes de la vie en société, ils seraient particulièrement réticents à avouer leurs délits. 
cet indice au niveau individuel, on additionne un point pour chacun des différents délits du sondage qui a été admis au moins une fois. Ainsi, un score de trois dans cet indice signifie que la personne a avoué avoir commis (au moins une fois) trois des délits contenus dans le sondage. Ensuite, on calcule la moyenne pour l'ensemble de l'échantillon ${ }^{4}$.

Les mêmes indices ont été construits à partir des interpellations enregistrées par la police. Dans ce cas, au lieu de calculer les indices à partir des délits avoués, on les calcule à partir des interpellations enregistrées par la police.

Les prochains paragraphes setont consacrés à diverses analyses qui devraient nous permettre d'établir le degré de validité des indicateurs comparés de la criminalité. Dans ce contexte, la validité d'un indicateur peut être définie comme son aptitude à mesurer efficacement la criminalité. Deux grands types de validité (et leurs sous-types respectifs) seront étudiés, à savoir la validité de contenu et la validité concourante. Finalement, signalons d'ores et déjà que les résultats présentés ici ont été contrôlés en prenant en considération le sexe et l'âge des membres de l'échantillon, sans que des différences significatives aient été trouvées.

\section{Validité de contenu}

Les difficultés liées à la validité de contenu ne se posent pas au niveau des données de police parce qu'en principe, dans un État de droit, la police n'interpelle une personne que lorsque cette dernière est suspectée d'avoir commis une infraction. Le contenu des dossiers de police est donc clair. En revanche, les sondages de délinquance autoreportée contiennent souvent des comportements qui ne suivent pas la logique des infractions à la loi pénale. Par conséquent, lors de l'analyse de la validité d'un sondage de délinquance autoreportée, il est opportun de contrôler si les questions du sondage concernent effectivement des comportements

4. Bien qu'il s'agisse d'une moyenne, l'indice de la diversité de la délinquance s'avère moins sensible aux valeurs extrêmes que l'indice de l'incidence. En effet, la valeur maximale possible pour l'indice de la diversité est de douze (ce qui correspond au nombre de délits inclus dans le sondage), tandis qu'au niveau de l'incidence, rien n'empêche une personne de soutenir qu'elle a commis plusieurs délits quotidiennement. Dans la pratique, ce dernier cas de figure ne s'est pas vérifié. D'autre part, le nombre maximum de délits différents (diversité de la délinquance) avoués par les membres de notre échantillon, pour la période de six mois avant l'admission dans le programme, a été de huit (voir graphique 4). 
délinquants et si elles représentent de manière adéquate l'univers des comportements étudiés, c'est-à-dire la délinquance.

Cette définition de la validité de contenu permet de montrer qu'elle cherche à répondre à deux questions différentes qui constituent en fait deux sous-types de validité : la validité apparente (les questions du sondage concernent-elles des comportements délinquants ?) et la validité logique (les questions du sondage représentent-elles de manière adéquate la délinquance ?).

La question de la validité apparente peut paraitre un non-sens aux yeux d'un profane. En effet, par définition, un sondage de délinquance autoreportée est censé mesurer la délinquance. Or, il est connu qu'entre autres défauts, les premiers sondages de délinquance autoreportée contenaient souvent des comportements problématiques, c'est-à-dire des comportements qui ne sont pas des délits mais plutôt des formes mineures de déviance disparaissant avec l'âge (par exemple, l'absentéisme scolaire ou les fugues) et des délits triviaux, c'est-à-dire des délits qui, à eux seuls, ne feraient presque jamais l'objet d'une procédure judiciaire, comme, par exemple, la resquille dans les transports publics ou les vols d'objets d'une très faible valeur (moins de 5 dollars américains). D'ailleurs, ce genre de comportements déviants a été fréquemment mélangé à des délits sérieux pour construire des échelles de la délinquance, ce qui s'explique par le fait que dans les années 1950 et 1960 on utilisait souvent l'échelle de Guttman, qui exige la présence d'une série de comportements pouvant être classés par ordre de gravité croissante.

Dans le tableau 1 , nous testons la validité apparente du sondage de délinquance autoreportée utilisé dans notre recherche, en comparant les comportements y inclus avec des infractions prévues dans la loi pénale. Étant donné que notre but n'est pas de faire une présentation orientée vers le droit pénal, nous avons préféré présenter les principales dispositions légales équivalentes, plutôt que d'entrer dans le détail de toutes les dispositions qui pourraient éventuellement être appliquées selon les circonstances de commission de chaque infraction en particulier.

Cette comparaison montre que le sondage ne contient ni des comportements problématiques ni des délits triviaux. De plus, on peut constater que le libellé des questions ne laisse pas de place à l'ambiguité 5 . On pourrait même parler d'un libellé aggravé, notamment dans le cas des lésions

5. Dans le tableau 1, nous présentons le libellé des questions concernant la prévalence " vie » des comportements (c'est-à-dire le fait d'avoir déjà commis tel ou tel délit au moins une fois). Les questions détaillées consécutives à une téponse affirmative permettaient d'établir la prévalence et l'incidence pour les six derniers mois (voir Aebi, 2000) 
TABLEAU 1

Correspondances entre les délits du sondage de délinquance autoreportée et la loi pénale*

\begin{tabular}{|c|c|c|c|}
\hline Code & Délit & Libellé de la question & $\begin{array}{c}\text { Principales } \\
\text { dispositions légales } \\
\text { équivalentes }\end{array}$ \\
\hline 0.100 & $\begin{array}{l}\text { Vol d'argent dans } \\
\text { un automate ou } \\
\text { une cabine } \\
\text { téléphonique }\end{array}$ & $\begin{array}{l}\text { Avez-vous déjà endommagê une } \\
\text { cabine téléphonique, un distributeur } \\
\text { de billets ou un autre type } \\
\text { d'automate à sous, pour y prendre } \\
\text { de l'argent? }\end{array}$ & $\begin{array}{l}\text { Vol (art. } 139 \text { CPS) } \\
\text { + dommages } \\
\text { à la propriété } \\
\text { (art. } 144 \text { CPS) }\end{array}$ \\
\hline D.110 & Vol à l'étalage & $\begin{array}{l}\text { Avez-vous déjà pris sans payer } \\
\text { quelque chose dans un magasin, } \\
\text { dans une boutique, dans une grande } \\
\text { surface ou dans un kiosque? }\end{array}$ & Val (art. 139 CPS) \\
\hline 0.120 & $\begin{array}{l}\text { Pickpocket } \\
\text { (vol à la tire) }\end{array}$ & $\begin{array}{l}\text { Avez-vous déjà fait les poches des } \\
\text { gens? (faire du pickpocket) }\end{array}$ & Vol (art. 139 CPS) \\
\hline D.130 & Vol à l'arraché & $\begin{array}{l}\text { Avez-vous déjà arraché à quelqu'un } \\
\text { son sac à main, son porte-monnaie } \\
\text { ou autre chose en vue de lui prendre } \\
\text { son argent? (faire du vol à l'arraché) }\end{array}$ & Vol (art. 139 CPS) \\
\hline D.140 & Cambriolage & $\begin{array}{l}\text { Avez-vous déjà pénétré sans } \\
\text { autorisation ou par effraction } \\
\text { [en forçant les portes ou fenêtres] } \\
\text { dans une maison, un appartement, } \\
\text { un bātiment ou un autre local pour y } \\
\text { voler quelque chose? }\end{array}$ & $\begin{array}{l}\text { Vol (art. } 139 \text { CPS) } \\
\text { + violation de domicile } \\
\text { (art. } 186 \text { CPS) } \\
\text { + éventuellement } \\
\text { dommages } \\
\text { à la propriété } \\
\text { (art. } 144 \text { CPS) }\end{array}$ \\
\hline D.150 & Recel & $\begin{array}{l}\text { Avez-vous déjà vendu quelque chose } \\
\text { dont vous saviez ou pensiez qu'elle } \\
\text { avait été volée? }\end{array}$ & Recel (art. 160 CPS) \\
\hline D. 200 & Brigandage & $\begin{array}{l}\text { Avez-vous déjà menacé quelqu'un } \\
\text { avec une arme ou frappé quelqu'un, } \\
\text { en vue de lui prendre son argent ou } \\
\text { d'autres choses de valeur? }\end{array}$ & $\begin{array}{l}\text { Brigandage } \\
\text { (art. } 140 \text { CPS) }\end{array}$ \\
\hline 0.210 & $\begin{array}{l}\text { Lésions } \\
\text { corporelles }\end{array}$ & $\begin{array}{l}\text { Avez-vous déjà frappé violemment } \\
\text { ou blessé quelqu'un, à tel point que } \\
\text { vous pensiez ou saviez qu'il a dû } \\
\text { recevoir une aide médicale ou des } \\
\text { soins d'un médecin? }\end{array}$ & $\begin{array}{l}\text { Lésions corporelles } \\
\text { graves ou simples } \\
\text { (arts } 122-123 \text { CPS) }\end{array}$ \\
\hline
\end{tabular}




\begin{tabular}{|l|l|l|l|} 
D.220 & Lésions avec arme & $\begin{array}{l}\text { Avez-vous déjà blessé } \\
\text { [intentionnellement] quelqu'un avec } \\
\text { un couteau, un bảton ou une autre } \\
\text { arme? [Ici, vous devez écarter les } \\
\text { comportements que vous avez déjà } \\
\text { mentionnés au numéro D.200 et } \\
\text { D.210] }\end{array}$ & $\begin{array}{l}\text { Lésions corporelles } \\
\text { simples aggravées } \\
\text { (art. 123 CPS) }\end{array}$ \\
\hline D.300 & $\begin{array}{l}\text { Trafic de drogues } \\
\text { douces }\end{array}$ & $\begin{array}{l}\text { Avez-vous déjà vendu du haschisch, } \\
\text { de la marijuana, du cannabis ? }\end{array}$ & $\begin{array}{l}\text { Trafic de substances } \\
\text { stupéfiants } \\
\text { (art. 19 Lstup) }\end{array}$ \\
\hline D.310 & $\begin{array}{l}\text { Trafic de drogues } \\
\text { dures }\end{array}$ & $\begin{array}{l}\text { Avez-vous déjà vendu certaines } \\
\text { drogues dures telles que l'héroïne, } \\
\text { cocaine, LSD, ecstasy, PCP, crack, } \\
\text { amphétamines, etc. ? }\end{array}$ & $\begin{array}{l}\text { Trafic de substances } \\
\text { stupéfiants } \\
\text { (art. 19 Lstup) }\end{array}$ \\
\hline D.320 & $\begin{array}{l}\text { Escroquerie dans } \\
\text { le trafic de drogue }\end{array}$ & $\begin{array}{l}\text { Avez-vous déjà arnaqué quelqu'un, } \\
\text { lorsque vous lui vendiez de la } \\
\text { drogue? }\end{array}$ & $\begin{array}{l}\text { Escroquerie } \\
\text { (art. 146 CPS) }\end{array}$ \\
\hline
\end{tabular}

*CPS : Code pénal suisse; LStup : Loi sur les stupéfiants

corporelles pour lesquelles on exige l'intervention d'un médecin. Dans ce contexte, les délits retenus ne pourraient pas être confondus avec des comportements légaux ou à la limite de la légalité. Il s'agit donc d'un questionnaire destiné à mesurer la délinquance et non les formes mineures de la déviance.

En ce qui concerne la validité logique, ces 12 délits ne recouvrent évidemment pas l'ensemble des délits prévus dans la loi pénale, mais plutôt les délits les plus fréquemment commis par des héroönomanes (Killias et Uchtenhagen, 1995). Ce choix s'explique par le fait que ces derniers devaient répondre au moins trois fois au sondage de délinquance autoreportée et qu'ils répondaient en même temps à d'autres questionnaires sur leur état de santé et leur situation économique et sociale. Par conséquent, il était nécessaire de développer un sondage relativement court. Néanmoins, le contrôle des registres de police indique qu'un éventuel élargissement des délits inclus dans le sondage afin de recouvrir l'ensemble de la délinquance connue des corps de police ne se traduirait que par une augmentation de moins de $10 \%$ des personnes identifiées comme délinquantes (voir le tableau 3 ciaprès). En effet, il n'y a que ce pourcentage de l'échantillon qui ait été interpellé par la police pour des délits autres que ceux prévus dans le sondage et qui n'ait pas en même temps avoué au moins l'un des délits 
du sondage. Une analyse plus détaillée montre que la plupart de ces interpellations concernent des infractions à la loi sur la circulation routière (qui n'ont pas été incluses dans le sondage, parce que la participation au programme exigeait le dépôt du permis de conduire), ainsi que des vols classés dans la catégorie " autres vols", pour laquelle on ne peut pas trouver de correspondance exacte au niveau du sondage de délinquance autoreportée.

\section{Validité concourante}

Dans le domaine des sciences de la nature, la validité concourante d'un indicateur est établie en comparant ses performances avec celles d'un autre indicateur dont la validité a déjà été prouvée. Dans la mesure où il sera capable de reproduire (ou d'améliorer) les performances obtenues avec l'indicateur utilisé comme patron pour la comparaison, on dira qu'il est valide par rapport à celui-là. Dans le domaine des sciences sociales, dont la criminologie fait partie, il y a malheureusement peu d'indicateurs dont la validité ait été prouvée. Pour cette raison, les comparaisons entre les indicateurs de criminalité chercheront à établir leur degré de corrélation ; mais, sachant que le plus souvent ces corrélations seront loin d'être parfaites, on essaiera de savoir si un indicateur permet au moins de distinguer des individus considérés comme différents par un autre indicateur.

Ainsi, les chercheurs en criminologie distinguent deux grands types de validité concourante : la validité pour groupes différents, dans laquelle on teste l'aptitude d'un instrument à distinguer des groupes censés être différents dans leur implication dans la délinquance d'après d'autres critères; et la validité corrélationnelle, dans laquelle on cherche à établir le degré de corrélation entre les différents indicateurs de la délinquance.

En ce qui concerne la validité pour groupes différents, l'indicateur habituellement testé est le sondage de délinquance autoreportée. À cet effet, on utilise un indicateur officiel de la délinquance (dans notre cas, les interpellations enregistrées par la police) pour constituer deux groupes :

a) délinquants : il s'agit des personnes qui ont été interpellées par la police durant la période considérée ;

b) non délinquants : il s'agit des personnes qui n'ont pas été interpellées par la police durant la période considérée. 
Ensuite, on compare les scores de ces deux groupes dans les indices établis à partir du sondage de délinquance autoreportée. La logique de la comparaison suppose que le groupe des délinquants aura un score plus élevé que le groupe des non-délinquants. Ceci est le cas dans notre recherche, où nous constatons (voir graphique 1) que le groupe des délinquants $(\mathrm{N}=223)$ a admis en moyenne 61 délits, tandis que le groupe de non-délinquants $(N=268)$ en a admis 30. De même, au niveau de la diversité de la délinquance autoreportée, le groupe de délinquants a un score de 1,8 alors que celui du groupe de non-délinquants est de 1,1 .

Comme le montrent les revues de la littérature de Huizinga et Elliot (1986), Fréchette et Le Blanc (1987), Junger-Tas (1989) et Junger-Tas et Marshall (1999), cette méthode en vue de tester la validité pour des

\section{GR A P H I Q U E 1}

Diversité et incidence de la délinquance autoreportée selon l'implícation dans la délinquance d'après les données de police

(période d'observation : 6 mois avant l'admission dans le programme)*

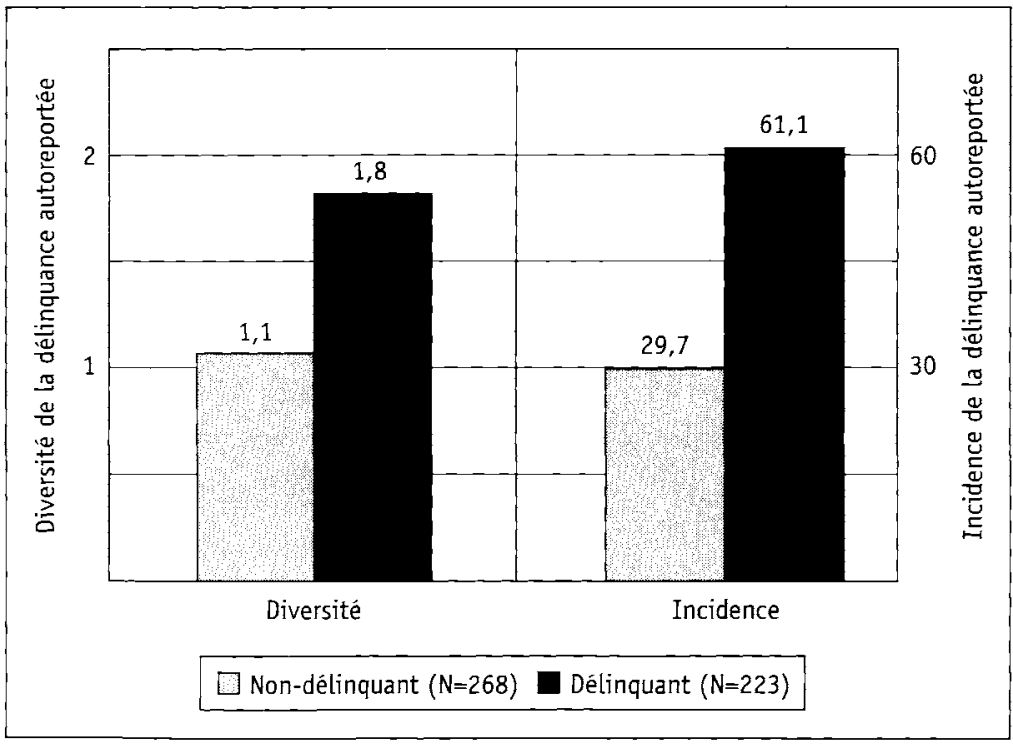

* Étant donné les différences de proportion dans les valeurs absolues de ces indices, nous avons utilisé deux axes d'ordonnées différentes. À gauche du graphique, l'axe est gradué d'après la diversité de la délinquance autoreportée; à droite, d'après l'incidence. 
groupes différents a été utilisée régulièrement par les chercheurs. Pourtant, ces detniers ont vite décelé qu'elle présentait un inconvénient de taille (voir Hindelang et al., 1981 : 97). En effet, cette méthode accepte dès le départ la validité des données de police. En fait, les sondages de délinquance autoreportée sont considérés comme valides parce qu'ils produisent des résultats semblables aux résultats obtenus avec les données de police. Mais la validité des indicateurs officiels de la délinquance (dont les données de police font partie) n'a jamais été prouvée. Bien au contraire, on a tendance à considérer que ces indicateurs ne sont pas des indicateurs de la criminalité, mais plutôt de la réaction sociale face au crime. Ainsi, Robert et al. (1994) considèrent qu'ils renseignent sur les politiques publiques de gestion de la délinquance, et Picca (2000 : 52) considère qu'ils «n'ont pas pour objet principal de nous renseigner sur l'état de la criminalité, mais davantage de mesurer l'activité des services qui les établissent : police ou justice. »

Pour cette raison, nous avons également testé la validité pour des groupes différents dans le sens inverse, c'est-à-dire en établissant les groupes à partir du sondage de délinquance autoreportée et en comparant ensuite leurs scores de diversité et d'incidence de la délinquance selon les données de police. Nous croyons que cette méthode n'a pas été utilisée par le passé parce qu'habituellement les sondages de délinquance autoreportée ont été menés auprès d'écoliers et de jeunes qui n'étaient pas très impliqués dans la délinquance (ou, à l'inverse, auprès de personnes incarcérées pour lesquelles il était a priori impossible d'établir un groupe de non-délinquants). Or, comme nous l'avons déjà vu, ceci n'est pas du tout le cas dans notre recherche. Au contraire, notre échantillon est composé d'adultes très impliqués dans la délinquance.

Cette méthode alternative pour tester la validité pour des groupes différents est intéressante, car elle permet :

a) d'établir partiellement la validité des données autoreportées. En effet, elle permet de découvrir si les personnes sondées cachent leur délinquance et, dans l'affirmative, de mesurer l'étendue de cette délinquance cachée. Ce cas de figure se présenterait si les personnes interpellées par la police n'admettaient pas des délits dans le sondage de délinquance autoreportée ;

b) d'établir partiellement la validité des données de police. En effet, elle permet de savoir si la police arrive ou non à identifier les personnes les plus impliquées dans la délinquance (c'est-à-dire celles qui admettent avoir commis des délits). 
GRAPHIQUE 2

Diversité et incidence des interpellations enregistrées par la police selon l'implication dans la délinquance d'après le sondage de délinquance autoreportêe (période d'observation : 6 mois avant l'admission dans le programme)

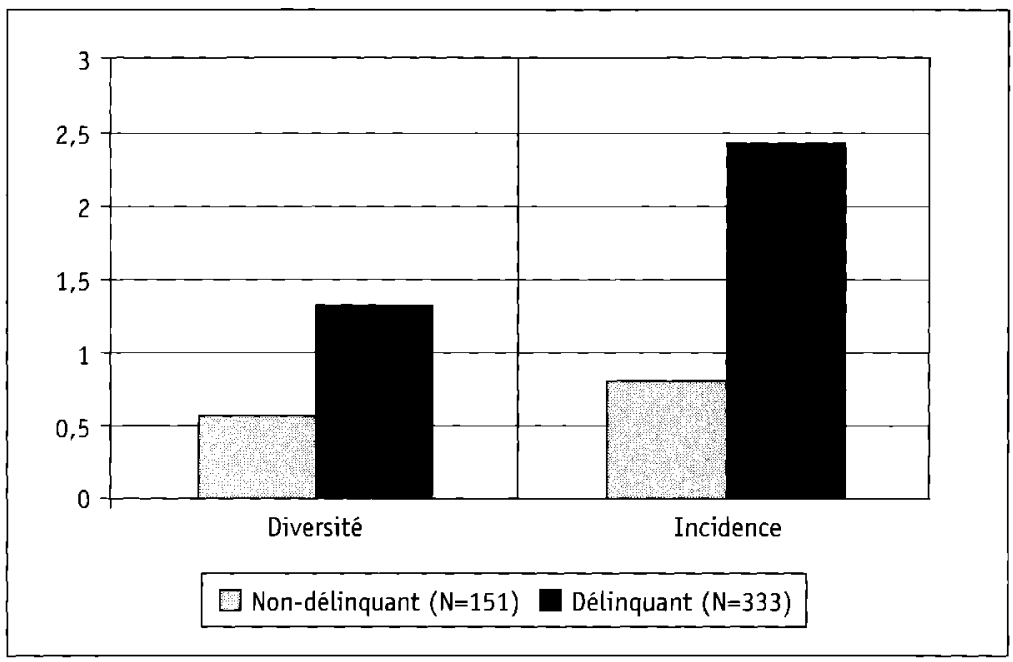

Naturellement, quelques discordances s'expliqueront du fait que certains délits menant à une interpellation enregistrée par la police n'ont pas pu être admis par les personnes sondées parce que le sondage de délinquance autoreportée ne contenait que douze délits (voir ci-dessus point 3).

Notre analyse (voir graphique 2) montre que le groupe de délinquants d'après le sondage de délinquance autoreportée $(\mathrm{N}=333)$ présente des scores plus élevés que le groupe de non délinquants $(\mathrm{N}=151)$. En effet, la police a enregistré une moyenne de 2,4 interpellations pour le premier groupe, contre 0,8 pour le deuxième, tandis qu'au niveau de la diversité de la délinquance enregistrée par la police, le rapport est de 1,3 contre 0,6. Ceci corrobore la validité des registres de police comme indicateurs de la criminalité. Par conséquent, nous pouvons dire, avec Fréchette et Le Blanc (1987: 81), que « l'action sélective du système de justice n'est pas si aléatoire et si discrétionnaire qu'on l'affirme généralement ». Dans le même sens, Farrington (1977) a signalé que la police ne semble pas choisir au hasard les personnes dont elle s'occupe. 
Notons également que la comparaison des graphiques 1 et 2 met en évidence des différences considérables quant au nombre absolu de délits enregistrés par les deux indicateurs comparés. Alors que dans le sondage, le nombre moyen de délits admis varie de 30 à 61 selon les groupes, la police a enregistré entre 0,8 et 2,4 interpellations. Ces différences soulignent l'importance du sondage en tant qu'instrument pour mesurer, au moins en partie, le chiffre noir de la délinquance.

À présent, il nous reste à établir la validité corrélationnelle, en calculant les corrélations entre les indicateurs comparés de la criminalité. Ce faisant (voir tableau 2), nous trouvons des corrélations positives et significatives, ce qui implique que les deux indicateurs mesurent le même concept. Étant donné que nous appelons ce concept la délinquance et qu'il s'agit du phénomène que nous voulions mesurer, nous pouvons considérer les deux indicateurs comme relativement valides, même si les corrélations ne sont pas fortes. Au niveau de l'incidence, la corrélation est de 0,26 lorsqu'on restreint l'analyse à des délits comparables (c'est-à-dire les douze délits retenus dans le sondage et leurs équivalents au niveau des registres de police) et de 0,30 lorsqu'on considère l'ensemble des interpellations enregistrées par la police. En ce qui concerne la diversité de la délinquance, les corrélations sont de 0,33 et 0,34 respectivement.

TABLEAU 2

Corrélations ( $r$ de Pearson) pour la diversité et l'incidence de la délinquance entre données autoreportées et données de police (période de référence : 6 mois avant l'admission dans le programme)

\begin{tabular}{|l|c|c|c|c|}
\hline \multirow{2}{*}{} & \multicolumn{2}{|c|}{ Diversité } & \multicolumn{2}{c|}{ Incidence } \\
\cline { 2 - 5 } & $\begin{array}{c}\text { Délits } \\
\text { comparables }\end{array}$ & $\begin{array}{c}\text { Tout type } \\
\text { de délit }\end{array}$ & $\begin{array}{c}\text { Délits } \\
\text { comparables }\end{array}$ & $\begin{array}{c}\text { Tout type } \\
\text { de délit }\end{array}$ \\
\hline$r$ de Pearson & 0,33 & 0,34 & 0,26 & 0,30 \\
& $(N=472)$ & $(N=472)$ & $(N=457)$ & $(N=457)$ \\
& $p: \leq 0,01$ & $p: \leq 0,01$ & $p: \leq 0,01$ & $p: \leq 0,01$ \\
\hline
\end{tabular}

En fait, ces corrélations sont comparables à celles qui ont été relevées par d'autres chercheurs (voir notamment les revues de la littérature de Hindelang et al., 1981 : ch. 5 ; de Huizinga et Elliott, 1986 ; et d'Aebi, 1999 : 142 ss.) et s'expliquent par un certain nombre de raisons, parmi lesquelles on peut citer :

a) la difficulté de trouver des correspondances exactes entre les délits retenus dans un sondage de délinquance autoreportée et les délits enregistrés par la police; 
b) les personnes très impliquées dans la délinquance ont de la peine à calculer le nombre exact de délits qu'elles ont commis et à les placer de manière adéquate dans le temps ;

c) un délinquant et un agent de police peuvent qualifier différemment le type de délit commis ;

d) certaines interpellations ne sont pas enregistrées de manière officielle par la police ;

e) les réponses des personnes sondées peuvent manquer d'honnêteté.

Dans la pratique, la question fondamentale est de savoir si les corrélations ne sont pas plus fortes à cause de la dernière de ces raisons, c'està-dire parce que les personnes sondées ont caché la totalité ou une partie de leur délinquance. Ce problème est d'autant plus aigu dans notre recherche que l'échantillon est composé de toxicomanes dont la plupart sont également des délinquants, soit deux catégories pour lesquelles il existe des doutes sérieux quant à la validité de leurs réponses (voir Brochu, 1995 : 211). Or, quand nous nous intéressons à la prévalence de la délinquance en comparant les personnes ayant admis au moins un délit dans le sondage avec les personnes interpellées par la police (voir tableau 3), nous pouvons voir que les divergences proviennent principalement des personnes qui ont admis des délits mais qui n'ont pas été interpellées par la police. En effet, en restreignant l'analyse à des délits comparables, on constate que $52 \%$ des personnes admettent des délits alors qu'elles n'ont pas été interpellées par la police, alors que seulement $2 \%$ ont été interpellées par la police et n'ont pas admis de délits. Lorsqu'on élargit l'analyse en considérant l'ensemble des interpellations enregistrées par la police, les pourcentages sont de $33 \%$ et $10 \%$ respectivement.

Afin de contrôler cette analyse, nous avons refait la comparaison entre les deux indicateurs de la criminalité après une année de traitement, et nous avons constaté que le résultat était à peu près identique. En effet, sur les 135 personnes ${ }^{6}$ qui avaient été traitées pendant au moins une année au moment de la collecte des données, $3 \%$ (4 personnes)

6. La collecte des données de police a commencé en septembre 1996 et, à ce momentlà, 135 des personnes sondées seulement avaient été traitées durant au moins douze mois (par exemple, toutes les personnes entrées dans un programme de ptescription d'héroïne durant l'année 1996 avaient une période d'observation inférieure à douze mois). Un contrôle des scores de ces personnes dans le sondage de délinquance autoreportée montre qu'elles ne se diffétencient pas de l'ensemble de l'échantillon ( $\mathrm{N}=491)$ que nous avons utilisé jusqu'à présent. 
TABLEAU 3

Implication dans la délinquance selon le sondage de délinquance autoreportée et les données de police pour délits comparables et pour tout type de délit ( $\mathrm{N}=484$; période de référence : 6 mois avant l'admission dans le programme)

\begin{tabular}{|l|c|c|c|c|}
\hline \multirow{2}{*}{$\begin{array}{l}\text { Implication dans } \\
\text { la délinquance }\end{array}$} & \multicolumn{2}{|c|}{ Délits comparables } & \multicolumn{2}{c|}{ Tout type de délit } \\
\cline { 2 - 5 } & $\mathrm{N}$ & $\%$ & $\mathrm{~N}$ & $\%$ \\
\hline $\begin{array}{l}\text { Non-délinquant d'après les deux } \\
\text { indicateurs }\end{array}$ & 141 & $29,1 \%$ & 104 & $21,5 \%$ \\
\hline $\begin{array}{l}\text { Délinquant d'après les deux } \\
\text { indicateurs }\end{array}$ & 82 & $16,9 \%$ & 174 & $36,0 \%$ \\
\hline $\begin{array}{l}\text { Délinquant d'après le sondage } \\
\text { uniquement }\end{array}$ & 251 & $51,9 \%$ & 159 & $32,8 \%$ \\
\hline $\begin{array}{l}\text { Délinquant d'après les données } \\
\text { de police uniquement }\end{array}$ & 10 & $2,1 \%$ & 47 & $9,7 \%$ \\
\hline Total & 484 & $100 \%$ & 484 & $100 \%$ \\
\hline
\end{tabular}

seulement ont été interpellées par la police et n'ont pas admis de délits comparables dans les sondages couvrant la première année de traitement (voir tableau 4). Ceci est d'autant plus surprenant que l'on aurait pu croire qu'à ce moment-là, les participants au programme auraient pu avoir tendance à avouer moins de délits pour donner une image positive des effets du traitement et en obtenir la poursuite. Or, il n'en est rien : ils continuent à avouer leurs délits, même si le nombre de ces derniers a enregistré une chute vertigineuse après l'admission dans le programme ${ }^{7}$.

7. Après une année de traitement, les corrélations entre les deux indicateurs pour l'indice de la diversité de la délinquance sont de 0,25 pour délits comparables $(\mathrm{p} \leq 0,01)$ et de 0,27 pour tout délit $(p \leq 0,01)$. En ce qui concerne l'incidence, elles sont de 0,23 pour délits comparables $(p \leq 0,01)$ et de 0,21 pour tout délit $(p \leq 0,05)$. 
TABLEAU 4

Implication dans la délinquance selon le sondage de délinquance autoreportée et les données de police pour délits comparables et pour tout type de délit ( $N=135$; période de référence : 12 mois après l'admission dans le programme)

\begin{tabular}{|l|c|c|c|c|}
\hline \multirow{2}{*}{$\begin{array}{l}\text { Implication dans } \\
\text { la délinquance }\end{array}$} & \multicolumn{2}{|c|}{ Délits comparables } & \multicolumn{2}{c|}{ Tout type de délit } \\
\cline { 2 - 5 } & $\mathrm{N}$ & $\%$ & $\mathrm{~N}$ & $\%$ \\
\hline $\begin{array}{l}\text { Non-dèlinquant d'après les deux } \\
\text { indicateurs }\end{array}$ & 67 & $49,6 \%$ & 50 & $37,0 \%$ \\
\hline $\begin{array}{l}\text { Délinquant d'après les deux } \\
\text { indicateurs }\end{array}$ & 10 & $7,4 \%$ & 23 & $17,0 \%$ \\
\hline $\begin{array}{l}\text { Délinquant d'après le sondage } \\
\text { uniquement }\end{array}$ & 54 & $40,0 \%$ & 41 & $30,4 \%$ \\
\hline $\begin{array}{l}\text { Délinquant d'après les données } \\
\text { de police uniquement }\end{array}$ & 4 & $3,0 \%$ & 21 & $15,6 \%$ \\
\hline Total & 135 & $100 \%$ & 135 & $100 \%$ \\
\hline
\end{tabular}

$\mathrm{Chi}^{2}$ : N.S. $(0,6) ;$ Phi : $0,16 \mathrm{Chi}^{2}$ : N.S. ; Phi : 0,07

\section{De la relation entre la délinquance autoreportée et les interpellations enregistrées par la police}

À première vue, les analyses que nous venons de présenter semblent confirmer que l'utilisation du sondage de délinquance autoreportée permet de découvrir une bonne partie de la délinquance cachée. Pourtant, on ne peut pas exclure que ces résultats soient dus à un problème de surreportabilité, phénomène qui se produit lorsqu'une personne admet des délits qu'elle n'a pas commis ${ }^{8}$. Heureusement, nous avons pu tester partiellement cette hypothèse, étant donné que nous avions demandé aux personnes sondées non seulement si elles avaient commis un délit, mais également si elles avaient été interpellées par la police à la

8. La surreportabilité peut se produire, entre autres, parce que les questions du sondage ont été rédigées de manière floue (Blackmore, 1974) ou parce qu'elles cherchent à dédramatiser ou à banaliser les délits inclus (ceci n'est pas le cas dans notre recherche, voir point 3 ci-dessus), mais également à cause du cadre dans lequel le sondage se déroule (dans un lycée, par exemple, un étudiant peut copier des réponses données par un camarade), ou par une incompréhension de la manière dont il faut remplir le questionnaire, ou bien par une combinaison de tous ces facteurs (Huizinga et Elliott, 1986). 
suite de ce délit. Ainsi, il a été possible de compater les interpellations admises par les personnes sondées avec les interpellations effectivement enregistrées par la police.

Sans entrer dans les détails de cette analyse, signalons d'ores et déjà qu'il est possible que certaines divergences entre les deux indicateurs s'expliquent soit parce que l'interpellation a été enregistrée par un corps

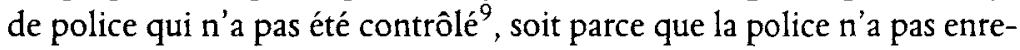
gistré de manière officielle l'interpellation; mais ces hypothèses ne pouvaient pas être testées avec les données à notre disposition. En revanche, nous avons constaté que le télescopage (phénomène qui se produit lorsqu'un individu considère qu'un événement s'est produit dans la période de référence alors qu'en réalité il s'est produit en dehors de cette période) est responsable de certaines divergences, et qu'une bonne partie de ces dernières s'expliquent par le fait que le délinquant et la police ont qualifié différemment le type de délit commis. Nous avons constaté, en particulier, que pas moins de 25 personnes qui ont admis avoir été interpellées pour trafic de drogues dures ont été enregistrées par la police pour possession ou consommation de drogues dures. On peut ainsi supposer que la personne a répondu au sondage de manière honnête en ce qui concerne ses intentions, mais qu'en règle générale, elle prend des précautions sur la quantité de drogue transportée afin d'éviter d'être accusée de trafic par la police. Il s'agit tout simplement d'une stratégie de survie pour des personnes qui ont désespérément besoin d'argent pour acheter des drogues.

Dans la même perspective, on pourrait penser que les personnes interpellées par la police à la suite de la commission d'un délit auront plus d'expérience sur la manière d'éviter ce gente d'interpellations dans le futur. Ce phénomène pourrait être encore plus marqué si la personne se spécialise dans la commission de certains délits.

Pour tester cette hypothèse nous avons établi le pourcentage de personnes interpellées par la police en fonction de l'incidence et de la diversité des délits que celles-ci avaient admis. En ce qui concerne l'incidence, nous avons tout d'abord regroupé les membres de l'échantillon dans des groupes d'environ 30 personnes, selon le nombre de délits

9. Rappelons que nous avons contrôlé les registres des dix corps de police des villes et des cantons dans lesquels se déroulait un programme de prescription d'héroine (et non pas l'ensemble des corps de police suisses). 
commis ${ }^{10}$. La seule exception est celle du groupe qui n'a pas avoué de délits, composé de 152 personnes. Un peu moins d'un tiers de ces dernières a toutefois été interpellé par la police, ce qui s'explique par le fait qu'au niveau de la police on prend en considération l'ensemble de la délinquance, tandis qu'au niveau de la délinquance autoreportée on ne considère que les douze délits retenus dans le sondage (il s'agit du $10 \%$ de l'échantillon dont nous avons parlé ci-dessus dans le point 3 ).

De manière prévisible, l'analyse montre (voir graphique 3) que la police interpelle plus fréquemment les personnes qui commettent un grand nombre de délits que celles qui en commettent peu ${ }^{11}$. Mais le

\section{GRAPHIQUE 3}

Pourcentage de personnes interpellées par la police selon l'incidence de la délinquance autoreportée

( $\mathrm{N}=457$; période de référence : 6 mois avant l'admission dans le programme)

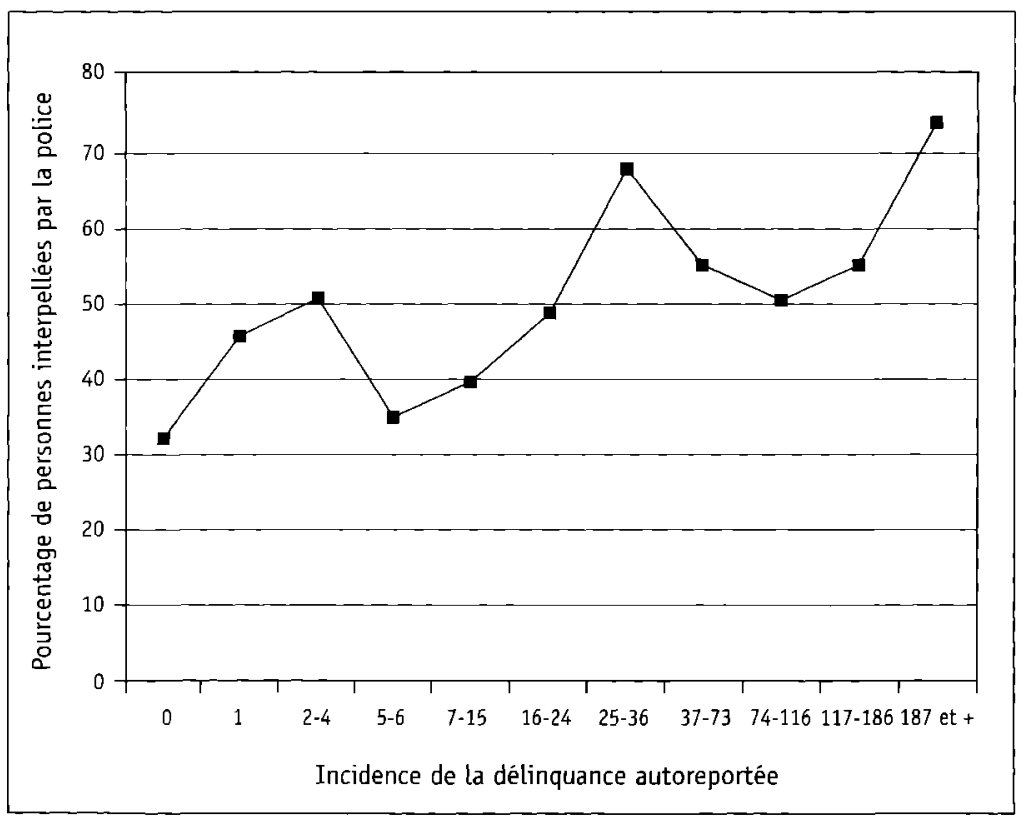

10. Signalons que d'auttes combinaisons, avec plus ou moins de personnes dans les groupes, mènent à des tésultats semblables à ceux présentés ici.

11. Cette hypothèse avait été postulée, avec de légères modifications de forme, par plusieurs chercheurs dont Erickson et Empey (1963) ; Dunford et Elliott (1984) ; Fréchette et Le Blanc (1987: 81) ; Fartington et al. (1996). 
rapport n'est pas linéaire, et un pourcentage considérable des personnes très impliquées dans la délinquance parvient à éviter les interpellations de la police. Ainsi, la police a interpellé $73 \%$ des personnes qui admettent avoir commis plus de 187 délits contre $45 \%$ de celles qui n'ont admis qu'un délit et $50 \%$ de celles qui en ont admis entre deux et quatre. Mais on constate également que ces pourcentages ne sont que de $50 \%$ pour les personnes qui ont commis entre 74 et 116 délits et de $54 \%$ pour celles qui en ont commis entre 117 et 186 .

En revanche, la diversité de la délinquance autoreportée (voir graphique 4) explique mieux les contacts avec la police. Ainsi, la police a interpellé $41 \%$ des personnes qui ont avoué un seul type de délit (soit 54 personnes sur 131), $54 \%$ des personnes qui ont avoué deux types de délit différents (soit 55 sur 102), $74 \%$ de celles qui en ont avoué quatre (soit 17 sur 23 ) et $100 \%$ de celles qui en ont avoué huit ( 2 personnes).

Cette tendance vers la spécialisation d'une partie considérable de l'échantillon pourrait s'expliquer par le profil particulier de ses membres.

\section{GRAPHIQUE 4}

Pourcentage de personnes interpellées par la police selon la diversité de la délinquance autoreportée

( $N=472$; période de référence : 6 mois avant l'admission dans le programme)

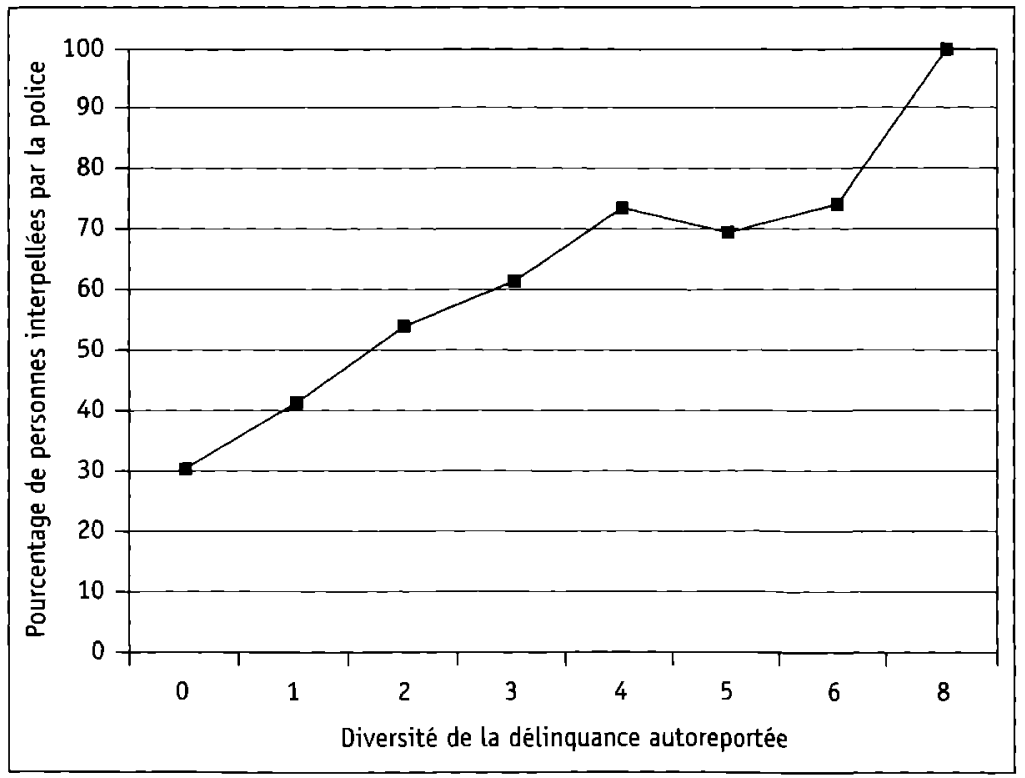


En effet, s'agissant de toxicomanes ayant besoin de commettre un grand nombre de délits pour payer quotidiennement leurs doses de stupéfiants, on pourrait imaginer qu'ils donnent la préférence à certains délits qui leur assurent une entrée régulière d'argent. On notera également que les toxicomanes ont un intérêt supplémentaire à éviter les contacts avec les autorités parce que, en les évitant, ils évitent en même temps le sevrage qui pourrait suivre une arrestation ou une incarcération.

La littérature sur ce sujet n'est pas univoque. En effet, il semble que certains toxicomanes se spécialisent dans une activité criminelle, tandis que d'autres commettent des délits de nature très variée (voir Brochu, 1995 : 32ss, avec références). Il semble également que l'implication dans la délinquance peut varier en fonction des différentes phases de la carrière d'un héroïnomane (voir Fauvel, 1991). Ainsi, la spécialisation criminelle se développerait lorsque le toxicomane arrive à la phase d'usage régulier d'héroüne mais qu'il peut encore mâ̂triser sa dépendance. En revanche, lorsque la dépendance est totale, le toxicomane ne pourrait alors plus planifier ses activités délinquantes et s'engagerait dans une gamme très large de délits (voir Brochu, 1995: 53ss, avec références).

Fauvel (1986) a également soutenu que c'est notamment lorsque le toxicomane s'éloigne de son activité délinquante spécifique (main bustle) qu'il se fait arrêter par les autorités. Les toxicomanes seraient ainsi arrêtés de manière disproportionnée pour des activités délinquantes « marginales ». Ceci a conduit cet auteur à remettre en question la validité des indicateurs officiels de la criminalité pour étudier la délinquance liée à la toxicomanie. Nos résultats ne nous permettent pas d'aller aussi loin. En effet, pour les toxicomanes de notre échantillon, les activités délinquantes principales sont les mêmes d'après les données de police et d'après les données autoreportées, à savoir le trafic de stupéfiants et les vols à l'étalage. Dans ce contexte, on ne peut pas écarter que le toxicomane interpellé par la police pour la commission d'un délit déterminé puisse avouer d'autres délits, ou que la police puisse le mettre en rapport avec la commission d'autres délits.

Il semble, en particulier, que cette tendance à la spécialisation n'ait pu être décelée qu'en raison de la courte période étudiée. En effet, lorsqu'on s'intéresse à la diversité des délits commis par l'ensemble des membres de l'échantillon $(\mathrm{N}=491)$ tout au long de leur vie, le score moyen est de 4,6. Ceci confirme la thèse déjà avancée par de nombreux chercheurs sur la versatilité des délinquants (Hirschi et Gottfredson, 
1995), c'est-à-dire la commission par ceux-ci d'un grand nombre de délits différents. Ce n'est que lorsqu'on restreint l'analyse aux six derniers mois que le score moyen descend à 1,4.

Ainsi, on pourrait imaginer que les délinquants très impliqués dans la criminalité (tels certains membres de notre échantillon) aient commis un nombre assez important de délits différents, mais que certains de ces délits aient été commis de manière occasionnelle et d'autres, avec une fréquence régulière. Ceci n'aurait rien d'étrange, vu qu'il s'agit d'un phénomène que l'on retrouve dans d'autres domaines de la vie quotidienne. Par exemple, un fumeur en manque peut accepter des cigarettes de n'importe quelle marque, ce qui ne l'empêche pas d'avoir une préférence pour une marque en particulier. En lui posant les bonnes questions, on découvrirait qu'il a fumé tout au long de sa vie des cigarettes de dizaines de marques différentes, mais qu'il a fumé davantage les cigarettes d'une certaine marque.

D'autre part, la diversité de la délinquance a un rapport avec la gravité des délits commis ${ }^{12}$. En effet, dans le cadre de notre sondage de délinquance autoreportée, on peut affirmer que la personne qui commet plusieurs délits commet forcément des délits graves, parce que ceux-ci sont majoritaires dans le questionnaire (voir tableau 1). Or, l'action de la police est fortement influencée par la gravité des délits commis, en ce sens qu'elle poursuit davantage les délits plus graves (par exemple, un cambriolage) que les délits moins graves (par exemple, un vol à l'étalage). Ainsi, bien que l'on ne puisse pas aisément placer les 12 délits du sondage dans un continuum allant du moins grave au plus grave, on peut considérer que la gravité des délits commis constitue une explication supplémentaire du rapport linéaire montré dans le graphique 4.

Dans cette perspective, l'importance de faire la distinction entre le court et le long terme réside dans le fait qu'elle permet d'expliquer de manière assez convaincante les contacts avec la police. D'après les résultats de notre recherche, bien que, en étudiant de longues périodes, on puisse constater que les délinquants qui ont commis le plus grand nombre de délits sont ceux qui ont été le plus souvent interpellés par la police, c'est cependant en étudiant des périodes plus courtes que l'on peut trouver une meilleure explication à ces interpellations. En effet, ce n'est pas tellement le nombre de délits commis qui détermine le risque

12. L'auteur remercie un lecteur anonyme pour sa réflexion fort intéressante sur la relation entre la diversité et la gravité de la délinquance. 
de se faire interpeller par la police, mais plutôt la diversité et la gravité de ces délits. Ceci confirme que les personnes qui se spécialisent dans la commission de certains délits tendent à développer des stratégies assez efficaces pour éviter les interpellations. Par contre, lorsqu'elles s'impliquent dans plusieurs types de délits différents, elles n'arrivent pas à maîtriser les techniques de commission de tous ces délits (" qui trop embrasse, mal étreint ", comme dit le proverbe) et finissent par se faire appréhender par la police ${ }^{13}$.

\section{Conclusion}

En guise de conclusion, nous pouvons dire que les registres de police, autant que les sondages de délinquance autoreportée, se sont avérés des indicateurs valides de la criminalité de l'échantillon étudié. Néanmoins, c'est le sondage de délinquance autoreportée qui nous a permis d'identifier le plus grand nombre de délits et de délinquants. Ajoutons que ce sont les personnes qui se définissent elles-mêmes comme délinquantes, mais qui n'ont pas été interpellées par la police, qui sont responsables de la faiblesse des corrélations entre ces deux indicateurs. Toutefois, pour avoir une image relativement complète de la criminalité, les registres de police sont le complément le plus adéquat du sondage. En effet, ils permettent de couvrir l'ensemble de la délinquance sans avoir à multiplier le nombre de délits inclus dans le sondage. En outre, combinés aux données autoreportées, ils permettent de tester des hypothèses très intéressantes en ce qui concerne le fonctionnement des forces de police. En particulier, nous avons pu constater que la gravité et la diversité de la délinquance expliquent, mieux que l'incidence, les contacts avec la police. Cette hypothèse est corroborée par le fait que, dans le court terme, ce sont les personnes qui s'engagent dans la gamme la plus large de délits (qu'elles ne peuvent pas tous maîtriser et qui dans tous les cas incluent des délits graves) qui sont davantage interpellées par la police; en revanche, une bonne partie de celles qui commettent un grand nombre de délits du même type arrivent à échapper aux forces de l'ordre.

13. Il serait intéressant de tester cette hypothèse avec d'autres échantillons parce qu'elle permettrait d'expliquer certaines « inconsistances » dans les carriètes délinquantes. Nous pensons, par exemple, aux délinquants sexuels qui ont des antécédents pour la commission de délits contre le patrimoine. 
Ce sont bien ces facteurs, et non pas le manque d'honnêteté dans les réponses des personnes sondées, qui justifient l'utilisation des registres de police. En effet, les personnes qui n'admettent pas des délits, tout en ayant été interpellées par la police pour des délits comparables aux délits du sondage, ne dépassent pas $3 \%$ de l'échantillon. Dans ce sens, soulignons que les conditions d'admission aux programmes de prescription d'héroïne exigeaient que les participants donnent leur accord pour que leurs antécédents policiers et judiciaires soient consultés et que les participants répondent au sondage de délinquance autoreportée. Néanmoins, cette obligation ne garantissait pas l'honnêteté des réponses données. Or, nos analyses confirment que les personnes sondées ont répondu de manière honnête. Ainsi, contrairement à ce que la recherche a montré pour des échantillons généraux de la population, le sondage de délinquance autoreportée peut être utilisé avec profit dans ce contexte particulier constitué de toxicomanes adultes très impliqués dans la délinquance, ayant eu des contacts avec le système de justice pénale et participant à un programme de prescription d'hérö̈ne.

\section{Références}

Aebi, M.F. (1999). La validité des indicateurs de la criminalité : Les sondages de délinquance autoreportée face aux données de police et du casier judiciaire. Thèse de doctorat, Lausanne : Université de Lausanne, Institut de police scientifique et de criminologie.

Aebi, M.F. (2000). Les indicateurs de la criminalité : leurs limitations, leur complémentarité et leur influence sur les théories criminologiques. Revue internationale de criminologie et de police technique et scientifique, 53 (2), 131 156.

Aebi, M.F., Ribeaud, D., \& Killias, M. (1999). Prescription médicale de stupéfiants et délinquance : Résultats des essais suisses. Criminologie, 32 (2), 127-148.

Blackmore, J. (1974). The relationship between self-reported delinquency and official convictions amongst adolescent boys. The British Journal of Criminology, 14 (2), 172-176.

Brochu, S. (1995). Drogue \& criminalité : Une relation complexe. Bruxelles : De Boeck Université / Ottawa : Presses de l'université d'Ottawa / Montréal : Presses de l'Université de Montréal.

Dunford, F.W., \& Elliott D.S. (1984). Identifying career offenders using selfreported data. Journal of Research in Crime and Delinquency, 21 (1), 57-86.

Erickson, M.L., \& Empey L.T. (1963). Court records, undetected delinquency and decision-making. The Journal of Criminal Law, Criminology and Police Science, 54, 456-469. 
Farrington, D.P. (1977). The effects of public labelling. The British Journal of Criminology, $17(2), 12-125$.

Farrington, D.P. (1989). Self-reported and official offending from adolescence to adulthood. In M. W. Klein (ed.), Cross-national research in selfreported crime and delinquency (pp.399-423). Dordrecht/Boston/London : Kluwer Academic Publishers.

Farrington, D.P., Loeber, R., Stouthamer-Loeber, M., van Kammen, W.B., \& Schmidt, L. (1996). Self-reported delinquency and a combined delinquency seriousness scale based on boys, mothers, and teachers : Concurrent and predictive validity for African-Americans and Caucasians. Criminology, $34(4), 493-517$

Faupel, C.E. (1986). Heroin use, street crime, and the « main hustle » : Implications for the validity of official crime data. Deviant Behavior, 7 (1), 31 45.

Faupel, C.E. (1991). Shooting dope : Career patterns of hard-core beroin users. Gainesville, FL : University of Florida Press.

Fréchette, M., \& Le Blanc M. (1987). Délinquances et délinquants. Chicoutimi : G. Morin.

Hirschi T., \& Gottfredson M.R. (1995). Control theory and the life-course perspective. Studies on Crime and Crime Prevention, 4 (2), 131-142.

Hindelang M.J., Hirschi T., \& Weis J.G. (1981). Medsuring delinquency Beverly Hills / London : Sage.

Huizinga, D., \& Elliott D.S. (1986). Reassessing the reliability and validity of self-report delinquency measures. Journal of Quantitative Criminology, 2 (4), 293-327.

Junger-Tas, J. (1989). Self-report delinquency research in Holland with a perspective on international comparison. In M. W. Klein (ed.), Cross-national research in self-reported crime and delinquency (pp.17-41). Dordrecht/ Boston/London : Kluwer Academic Publishers.

Junger-Tas, J., \& Marshall I.H. (1999). The self-report methodology in crime research. Crime and Justice : A Review of Research, 25, 291-367.

Killias, M. (2000). Problèmes éthiques en matière de recherches criminologiques : Les effets ironiques de la lutte contre les abus. In M. Killias (ed.), L'éthique et le droit (pp.299-312). Fribourg : Editions Universitaires Fribourg Suisse.

Killias, M., Aebi, M.F., Ribeaud, D., \& Rabasa, J. (1999). Rapport final sur les effets de la prescription de stupéfiants sur la délinquance des toxicomanes : Établi sur la base de données autoreportées, policières et du casier judiciaire, $2^{\mathrm{e} m e}$ éd. élargie. Lausanne : Université de Lausanne, Institut de police scientifique et de criminologie.

Killias, M., \& Uchtenhagen, A. (1995). Méthodologie de l'évaluation des essais suisses avec prescription médicale d'opiacés sous l'angle de la délinquance: L'accès sous contrôle médical à l'héroïne réduit-il la délinquance des toxicomanes? Bulletin de Criminologie, 21 (2), 33-48. 
Picca, G. (2000). La criminologie, $5^{e}$ éd. mise à jour. Paris : Presses Universitaires de France.

Reuband, K.-H. (1989). On the use of self-reports in measuring crime among adults : Methodological problems and prospects. In M.W. Klein (ed.), Cross-national research in self-reported crime and delinquency (pp.89-106). Dordrecht/Boston/London : Kluwer Academic Publishers.

Robert, P., Aubusson de Cavarlay, B., Pottier, M.-L., \& Tournier, P. (1994). Les comptes du crime: Les délinquances en France et leurs mesures, $2^{\text {êrne }}$ éd. refondue. Paris : L'Harmattan. 